\title{
END-TO-END FEEDBACK LOSS IN SPEECH CHAIN FRAMEWORK VIA STRAIGHT-THROUGH ESTIMATOR
}

\author{
Andros Tjandra ${ }^{1,2}$, Sakriani Sakti ${ }^{1,2}$, Satoshi Nakamura ${ }^{1,2}$ \\ ${ }^{1}$ Nara Institute of Science and Technology, Japan \\ ${ }^{2}$ RIKEN, Center for Advanced Intelligence Project AIP, Japan \\ \{andros.tjandra.ai6, ssakti, s-nakamura\}@is.naist.jp
}

\begin{abstract}
The speech chain mechanism integrates automatic speech recognition (ASR) and text-to-speech synthesis (TTS) modules into a single cycle during training. In our previous work, we applied a speech chain mechanism as a semi-supervised learning. It provides the ability for ASR and TTS to assist each other when they receive unpaired data and let them infer the missing pair and optimize the model with reconstruction loss. If we only have speech without transcription, ASR generates the most likely transcription from the speech data, and then TTS uses the generated transcription to reconstruct the original speech features. However, in previous papers, we just limited our back-propagation to the closest module, which is the TTS part. One reason is that backpropagating the error through the ASR is challenging due to the output of the ASR are discrete tokens, creating non-differentiability between the TTS and ASR. In this paper, we address this problem and describe how to thoroughly train a speech chain end-toend for reconstruction loss using a straight-through estimator (ST). Experimental results revealed that, with sampling from ST-Gumbel-Softmax, we were able to update ASR parameters and improve the ASR performances by $11 \%$ relative CER reduction compared to the baseline.
\end{abstract}

\section{Introduction}

A speech chain [1] is a viewpoint that describes the speech communication process in which the speaker produces words and generates speech sound waves, transmits the speech waveform through a medium (i.e., air), and creates a speech perception process in a listeners auditory system to perceive what was said. The hearing process is critical, not only for the listener but also for the speaker herself. By simultaneously listening and speaking, the speaker can monitor her volume, articulation, and the general comprehensibility of her speech. Based on those observations, we simulated the speech chain mechanism by coupling ASR and TTS and formed a machine speech chain [2, 3], so that the machine can learn, not only to listen (by way of ASR) or speak (by way of TTS) but also listen while speaking.

In our previous paper 2, we utilized the speech chain idea for semi-supervised learning using paired and unpaired data. First, we pretrained both ASR and TTS with a small amount of paired speech and text data. Then, we subsequently used both the pretrained modules to complete the missing pair from the unpaired data. For example, if we only have speech without transcription, ASR generates the most likely transcription from the speech data with greedy or beam-search decoding, and TTS uses the generated transcription to reconstruct the original speech features. In this case, we trained the TTS module with the reconstruction loss. For the reverse 
case, if we only have text without any corresponding speech, TTS generates speech, whose features ASR uses to reconstruct the original text. In this case, we updated the ASR module with the reconstruction loss. In Fig. 1(a), we illustrate a multispeaker speech chain loop between the ASR and TTS modules.

However, the auditory feedback in a human speech chain happens almost constantly, not only during semi-supervised learning. Furthermore, the closeloop feedback is also done end-to-end. But, to simulate our speech chain mechanism to provide the ability to help each other even during the supervised learning and perform a completely end-to-end feedback reconstruction loss, the main challenge is to utilize TTS to improve our ASR module. One reason is that back-propagating the error from the reconstruction loss through the ASR module is challenging due to the output of the ASR discrete tokens (grapheme or phoneme), creating non-differentiability between the TTS and ASR modules (Fig. 1(b)).

We address this problem using a straight-through estimator [4, 5. to predict the gradient through discrete variables (Fig. 1(c)). We mainly focus on describing how to thoroughly train a speech chain endto-end by adding a reconstruction term from the TTS module and backpropagated the gradient through the ASR. Experimental results revealed that, with teacher-forcing and sampling from Gumbel-Softmax, we were now able to updated ASR parameters and improved the ASR performances significantly by $11 \%$ relative CER reduction compared to the baseline.

\section{Speech Chain and End-to- end Feedback Loss}

In the speech chain mechanism, given speech features $\mathbf{x}=\left[x_{1}, . ., x_{S}\right]$ (e.g., Mel-spectrogram) and $\mathbf{y}=\left[y_{1}, . ., y_{T}\right]$, we feed the speech to the ASR module, and the ASR decoder generates continuous vector $h_{t}^{d}$ step-by-step. To calculate probability vector $\mathbf{p}_{y}=\left[p_{y_{1}}, . ., p_{y_{T}}\right]$, we apply the softmax function $p_{y_{t}}=\operatorname{softmax}\left(h_{t}^{d}\right)$ to decoder output $h_{t}^{e}$. For each class probability mass in $p_{y_{t}}, p_{y_{t}}[c]$ was defined as:

$$
p_{y_{t}}[c]=\frac{\exp \left(h_{t}^{d}[c] / \tau\right)}{\sum_{i=1}^{C} \exp \left(h_{t}^{d}[i] / \tau\right)}, \quad \forall c \in[1 . . C]
$$

Here $C$ is the total number of classes, $h_{t}^{d} \in \mathbb{R}^{C}$ are the logits produced by the last decoder layer, and $\tau$ is the temperature parameters. Setting temperature $\tau$ using a larger value $(\tau>1)$ produces a smoother probability mass over classes [6].

For the generation process, we generally have two different methods:

1. Conditional generation given ground-truth (teacher-forcing):

If we have paired speech and text $(\mathbf{x}, \mathbf{y})$, we can generate $p_{y_{t}}$ from autoregressive ASR decoder $D e c_{A S R}$, conditioned to ground-truth text $y_{t-1}$ in the current time-step and encoded speech feature $\mathbf{h}^{e}$. At the end, the length of probability vector $\mathbf{p}_{y}$ is fixed to $T$ time-steps.

2. Conditional generation given previous step model prediction:

Another generation process to decode ASR transcription uses its own prediction to generate probability vector $p_{y_{t}}$. There are many different generation methods, such as greedy decoding (1best beam-search) $\left(\tilde{y}_{t}=\underset{c}{\operatorname{argmax}} p_{y_{t}}[c]\right)$, beamsearch, or stochastic sampling $\left(\tilde{y}_{t} \sim \operatorname{Cat}\left(p_{y_{t}}\right)\right)$.

After the generation process, we obtained probability vector $\mathbf{p}_{y}$ and applied discretization from continuous probability vector $p_{y_{t}}$ to $\tilde{y}_{t}$ either by taking the class with the highest probability or sampling from a categorical random variable. After getting a single class to represent the probability vector, we encode it into vector $[0,0, \ldots, 1, . ., 0]$ with one-hot encoding representation and give it to the TTS as the encoder input. The TTS reconstructs Mel-spectrogram $\hat{\mathbf{x}}$ with the teacher-forcing approach. The reconstruction loss is calculated:

$$
\mathcal{L}_{T T S}^{r e c}=\frac{1}{S} \sum_{s=1}^{S}\left(x_{s}-\hat{x}_{s}\right)^{2}
$$




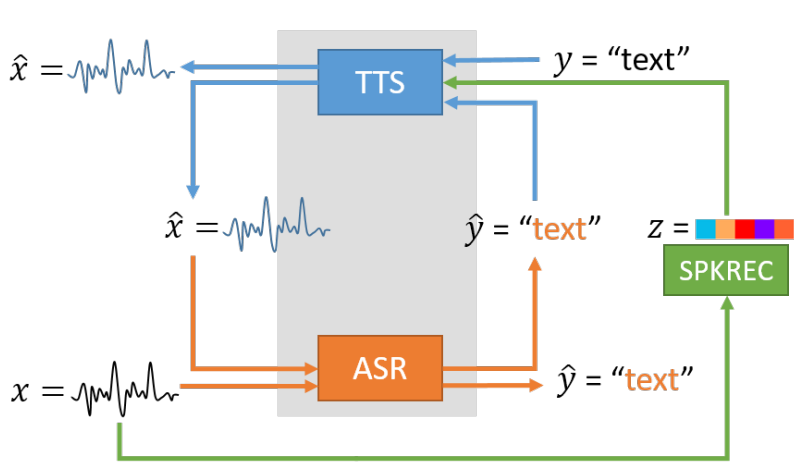

(a)

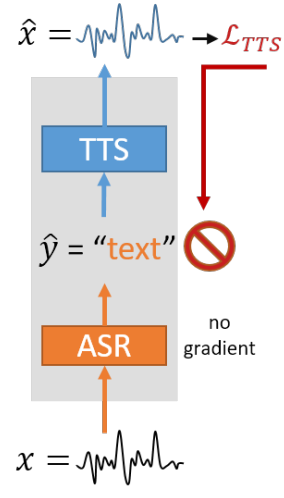

(b)

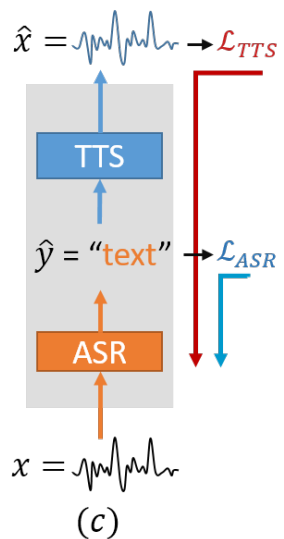

(c)

Figure 1: a) Multispeaker machine speech chain mechanism; b) Baseline ([3]): feedback loss from TTS is only backpropagated through the TTS module, and the ASR module is not updated because variable $\hat{y}$ is non-differentiable; c) Proposal: feedback loss from TTS is backpropagated through discrete variable $\hat{y}$, and ASR modules are updated based on the estimated gradient from the TTS module by a straight-through estimator.

where $\hat{x}_{s}$ is the predicted (or reconstructed) Melspectrogram and $x_{s}$ is the ground-truth spectrogram at $s$-th time-step.

We directly calculated the gradient from the reconstruction loss w.r.t TTS parameters $\left(\partial \mathrm{E}_{T T S}^{r e c} / \partial \theta_{T T S}\right)$ because all the operations inside the TTS module are continuous and differentiable. However, we could not calculate the gradient from the reconstruction loss w.r.t ASR parameters $\left(\partial \mathrm{E}_{T T S}^{r e c} / \partial \theta_{A S R}\right)$ because we have a discretization operation from $p_{y_{t}} \rightarrow$ $\operatorname{onehot}\left(\tilde{y}_{t}\right)$. Therefore, we applied a straight-through estimator to enable the loss from $\mathrm{E}_{T T S}^{r e c}$ to pass through discrete variable $\tilde{y}_{t}$.

\subsection{Straight-through Argmax}

The straight-through estimator [4, 5] is a method for estimating or propagating gradients through stochastic discrete variables. Its main idea is to backpropagate through discrete operations (e.g., $\underset{c}{\operatorname{argmax}} p_{y_{t}}[c]$ or sampling $\left.\tilde{y}_{t} \sim \operatorname{Cat}\left(p_{y_{t}}\right)\right)$ like an identity function. We describe the forward process and the gradient calculation with a straight-through estimator in Fig. 2.
In the implementation, we created a function with different forward and backward operations. For argmax one-hot encoding function, we formulated the forward operation:

$$
\begin{gathered}
\tilde{z}_{t}=\underset{c}{\operatorname{argmax}} p_{y_{t}}[c] \\
\tilde{y}_{t}=\operatorname{onehot}\left(\tilde{z}_{t}\right) .
\end{gathered}
$$

Here we describe $\tilde{y}_{t}$ as a one-hot encoding vector with the same length as the $p_{y_{t}}$ vector. When the loss is calculated and the gradients are backpropagated from loss $\mathcal{L}_{T T S}^{r e c}$, we formulate the backward operation:

$$
\frac{\partial \tilde{y}_{t}}{\partial p_{y_{t}}} \approx \mathbb{1}
$$

Therefore, when we back-propagate the loss from Eq. 2 with the straight-through estimator approach, we calculate the TTS reconstruction loss gradient 


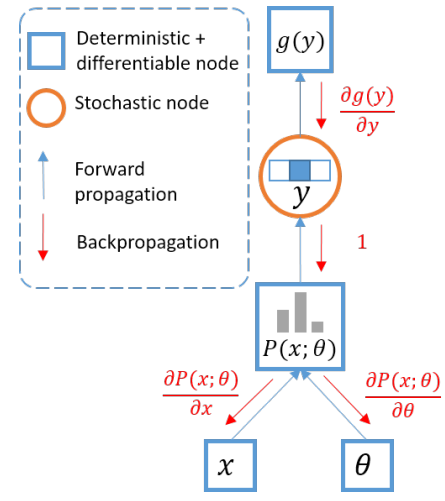

Figure 2: Straight-through estimator on $\arg \max$ function. Given input $x$ and model parameters $\theta$, we calculate categorical probability mass $P(x ; \theta)$ and apply discrete operation argmax. In the backward pass, the gradient from stochastic node $y$ to $P(x ; \theta)$, $\partial y / \partial P(x ; \theta) \approx \mathbb{1}$ is approximated by identity.

w.r.t $\theta_{A S R}$ :

$$
\begin{aligned}
\frac{\partial \mathcal{L}_{T T S}^{r e c}}{\partial \theta_{A S R}} & =\sum_{t=1}^{T} \frac{\partial \mathcal{L}_{T T S}^{r e c}}{\partial \tilde{y}_{t}} \cdot \frac{\partial \tilde{y}_{t}}{\partial p_{y_{t}}} \cdot \frac{\partial p_{y_{t}}}{\partial \theta_{A S R}} \\
& \approx \sum_{t=1}^{T} \frac{\partial \mathcal{L}_{T T S}^{r e c}}{\partial \tilde{y}_{t}} \cdot \mathbb{1} \cdot \frac{\partial p_{y_{t}}}{\partial \theta_{A S R}} .
\end{aligned}
$$

\subsection{Straight-through Gumbel Soft- $\max$}

Besides taking argmax class from probability vector $p_{y_{t}}$, we also generated a one-hot encoding by sampling with the Gumbel-Softmax distribution 7, 8. Gumbel-Softmax is a continuous distribution that approximates categorical samples, and the gradients can be calculated with a reparameterization trick. For Gumbel-Softmax, we replaced the softmax formula for calculating $p_{y_{t}}$ (Eq. 1):

$$
p_{y_{t}}[c]=\frac{\exp \left(\left(h_{t}^{d}[c]+g_{c}\right) / \tau\right)}{\sum_{i=1}^{C} \exp \left(\left(h_{t}^{d}[i]+g_{i}\right) / \tau\right)}, \quad \forall c \in[1 . . C] .
$$

where $g_{1}, . ., g_{C}$ are i.i.d samples drawn from Gum$\operatorname{bel}(0,1)$ and $\tau$ is the temperature. We sample $g_{i}$ by drawing samples from the uniform distribution:

$$
\begin{gathered}
u_{c} \sim \operatorname{Uniform}(0,1) \\
g_{c}=-\log \left(-\log \left(u_{c}\right)\right), \quad \forall c \in[1 . . C] .
\end{gathered}
$$

To generate a one-hot encoding, we define our forward operation:

$$
\begin{gathered}
\tilde{z}_{t} \sim \text { Categorical }\left(p_{y_{t}}[1], p_{y_{t}}[2], \ldots, p_{y_{t}}[C]\right) \\
\tilde{y}_{t}=\operatorname{onehot}\left(\tilde{z}_{t}\right) .
\end{gathered}
$$

At the backpropagation time, we use the same straight-through estimator (Eq. 5) to allow the gradients to flow through the discrete sampling operation from Eq. 11 .

\subsection{Combined Loss for ASR}

Our final loss function for ASR is a combination from negative likelihood (Eq. 16) and TTS reconstruction loss (Eq. 2) by sum operation:

$$
\mathrm{E}_{A S R}^{F}=\mathrm{E}_{A S R}+\mathrm{E}_{T T S}^{r e c} .
$$

To summarize our explanation in this section, we provide an illustration in Fig. 3 that explains how sub-losses $\mathrm{E}_{A S R}$ and $\mathrm{E}_{T T S}^{r e c}$ are backpropagated to the rest of the ASR and TTS modules.

\section{Sequence-to-Sequence Model for ASR}

A sequence-to-sequence model is a neural network that directly models conditional probability $p(y \mid x)$, where $\mathbf{x}=\left[x_{1}, \ldots, x_{S}\right]$ is the sequence of the (framed) speech features with length $S$ and $\mathbf{y}=\left[y_{1}, \ldots, y_{T}\right]$ is the labels sequence with length $T$.

The encoder task processes input sequence $x$ and generating representative information $h^{e}=$ $\left[h_{1}^{e}, \ldots, h_{S}^{e}\right]$ for the decoder. The attention module is an extension scheme that assists the decoder to find relevant information on the encoder side based on the 


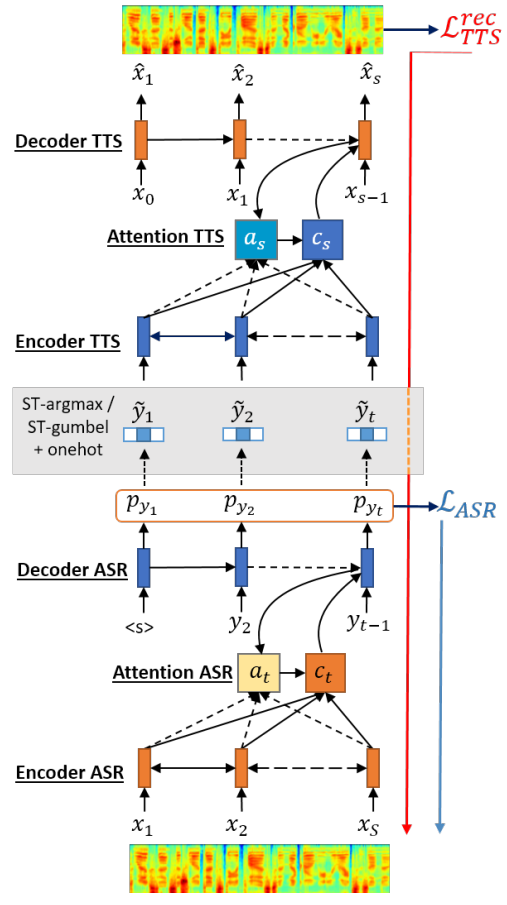

Figure 3: Given speech feature $\mathbf{x}$, ASR generates a sequence of probability $\mathbf{p}_{y}=\left[p_{y_{1}}, p_{y_{2}}, \ldots, p_{y_{T}}\right]$. If we have a ground-truth transcription, we can calculate $\mathrm{E}_{A S R}$ (Eq. 16). TTS module generates speech features, and we calculate reconstruction loss $\mathrm{E}_{T T S}^{r e c}$ (Eq. 2). After that, the gradients based on $\mathrm{E}_{A S R}$ are propagated through the ASR module, and the gradients based on $\mathrm{E}_{T T S}^{r e c}$ are propagated through the TTS and ASR modules by a straight-through estimator.

current decoder hidden states 9, 10. Attention modules produce context information $c_{t}$ at time $t$ based on the encoder and decoder hidden states:

$$
\begin{aligned}
c_{t} & =\sum_{s=1}^{S} a_{t}(s) * h_{s}^{e} \\
a_{t}(s) & =\operatorname{Align}\left(h_{s}^{e}, h_{t}^{d}\right) \\
& =\frac{\exp \left(\operatorname{Score}\left(h_{s}^{e}, h_{t}^{d}\right)\right)}{\sum_{s=1}^{S} \exp \left(\operatorname{Score}\left(h_{s}^{e}, h_{t}^{d}\right)\right)} .
\end{aligned}
$$

There are several variations for score functions [1] such as $S \operatorname{core}\left(h_{s}^{e}, h_{t}^{d}\right)$ : dot product $\left(\left\langle h_{s}^{e}, h_{t}^{d}\right\rangle\right)$, bilinear $\left(h_{s}^{e} \boldsymbol{\top} W_{s} h_{t}^{d}\right)$, where score : $\left(\mathbb{R}^{M} \times \mathbb{R}^{N}\right) \rightarrow \mathbb{R}, M$ is the number of hidden units for the encoder and $N$ is the number of hidden units for the decoder. Finally, the decoder task predicts target sequence probability $p_{y_{t}}$ at time $t$ based on previous output and context information $c_{t}$. The loss function for ASR can be formulated:

$$
\mathrm{E}_{A S R}=-\frac{1}{T} \sum_{t=1}^{T} \sum_{c=1}^{C} \mathbb{1}\left(y_{t}=c\right) * \log p_{y_{t}}[c],
$$

where $C$ is the number of output classes. Input $x$ for the speech recognition tasks is a sequence of feature vectors like a log Mel-scale spectrogram. Therefore, $x \in \mathbb{R}^{S \times D}$, where $\mathrm{D}$ is the number of features and $\mathrm{S}$ is the total frame length for an utterance. Output $y$, which is a speech transcription sequence, can be either a phoneme or a grapheme (character) sequence.

\section{Sequence-to-Sequence Model for TTS}

Speech synthesis can be viewed as a sequence-tosequence task where a model generates speech given a sentence. We directly model the conditional probability $p(x \mid y)$ with a sequence-to-sequence model, where $y=\left[y_{1}, \ldots, y_{T}\right]$ is the sequence of characters with length $T$ and $x=\left[x_{1}, \ldots, x_{S}\right]$ is the sequence of (framed) speech features with length $S$. From the sequence-to-sequence ASR model perspective, TTS is the reverse case where the model reconstructs the original speech given the text.

In this work, our core architecture is based on Tacotron 12 with several structural modifications 3]. The main difference between our modified Tacotron and the default Tacotron is that we added an additional speaker embedding projection layer into our decoder to enable multispeaker training and generation. We also have an additional output layer to generate binary prediction $b_{s} \in[0,1]$ ( 1 if the $s$-th frame is the end of speech, otherwise 0 ). 
For training the TTS model, we used the following loss function:

$$
\begin{aligned}
\mathrm{E}_{T T S}= & \frac{1}{S} \sum_{s=1}^{S}\left(x_{s}^{M}-\hat{x}_{s}^{M}\right)^{2}+\left(x_{s}^{R}-\hat{x}_{s}^{R}\right)^{2} \\
& -\left(b_{s} \log \left(\hat{b}_{s}\right)+\left(1-b_{s}\right) \log \left(1-\hat{b}_{s}\right)\right),
\end{aligned}
$$

where $\hat{x}^{M}, \hat{x}^{R}$, and $\hat{b}$ are the predicted log Mel-scale spectrogram, the log magnitude spectrogram, and the end-of-frame probability, and $x^{M}, x^{R}, b$ is the ground-truth. In the decoding process, we use the Griffin-Lim algorithm 13 to iteratively estimate the phase spectrogram and reconstruct the signal with inverse STFT.

\section{Experiment}

\subsection{Dataset}

We evaluated the performance of our proposed method on the Wall Street Journal dataset [14]. Our settings for the training, development, and test sets are the same as the Kaldi s5 recipe [15. We trained our model with WSJ-SI284 data. Our validation set was dev_93, and our test set was eval_92.

We used the character sequence as our decoder target and followed the preprocessing steps proposed by a previous work [16]. The text from all the utterances was mapped into a 32-character set: 26 (a-z) letters of the alphabet, apostrophes, periods, dashes, space, noise, and "eos." In all the experiments, we extracted the 40 dims $+\Delta+\Delta \Delta$ (total 120 dimensions) log Mel-spectrogram features from our speech and normalized every dimension into zero mean and unit variance.

\subsection{Model Details}

For the ASR model, we used a standard sequence-tosequence model with an attention module (Section 3). On the encoder sides, the input log Mel-spectrogram features were processed by three bidirectional LSTMs (Bi-LSTM) with 256 hidden units for each LSTM: a total of 512 hidden units for the Bi-LSTM. To reduce the memory consumption and processing time, we used hierarchical sub-sampling [17, 18] on all three Bi-LSTM layers and reduced the sequence length by a factor of eight. On the decoder sides, we projected one-hot encoding from the previous character into a 256-dims continuous vector with an embedding matrix, followed by one unidirectional LSTM with 512 hidden units. For the attention module, we used the content-based attention + multiscale alignment (denoted as "Att MLP-MA") 19 with a 1-history size. In the decoding phase, the transcription was generated by beam-search decoding $(\operatorname{size}=5)$, and we normalized the log-likelihood score by dividing it with its own length to prevent the decoder from favoring shorter transcriptions. We did not use any language model or lexicon dictionary in this work. In the training stage, we tried ST-argmax (Section 2.1) and ST-gumbel softmax (Section 2.2). We also tried both teacher-forcing and greedy decoding to generate ASR probability vectors $\mathbf{p}_{y}$. For each scenario, we treated temperature $\tau=[0.25,0.5,1,2]$ as our hyperparameter and searched for the best temperature based on the CER (character error rate) on the development set.

For the TTS model, we used the TTS explained in Section 4. The hyperparameters for the basic structure are generally the same as those for the original Tacotron, except we replaced ReLU with the LReLU function. For the $\mathrm{CBHG}$ module, we used $K=8$ filter banks instead of 16 to reduce the GPU memory consumption. For the decoder sides, we deployed two LSTMs instead of a GRU with 256 hidden units. For each time-step, our model generated four consecutive frames to reduce the number of steps in the decoding process.

\subsection{Experiment Result}

For our baseline, we trained an encoder-decoder with MLP + multiscale alignment with a 1-history size [19]. We also added several published results to our baseline. All of the baseline models were trained by minimizing negative log-likelihood $\mathrm{E}_{A S R}$ (Eq. 16).

All the models in the proposed section were trained with a combination from two losses: $\mathrm{七}_{A S R}+\mathrm{七}_{T T S}^{r e c}$, 
Table 1: ASR experiment result on WSJ dataset test_eval92.

\begin{tabular}{|c|c|c|c|}
\hline \multicolumn{4}{|c|}{ Baseline $\left(\mathbf{E}_{A S R}\right)$} \\
\hline \multicolumn{3}{|c|}{ Model } & CER (\%) \\
\hline \multicolumn{3}{|c|}{ Att MLP [20] } & 11.08 \\
\hline \multicolumn{3}{|c|}{ Att MLP + Location [20] } & 8.17 \\
\hline \multicolumn{3}{|c|}{ Att MLP [21] } & 7.12 \\
\hline \multicolumn{3}{|c|}{ Att MLP-MA (ours) 19} & 6.43 \\
\hline \multicolumn{4}{|c|}{ Proposed $\left(\mathbf{E}_{A S R}+\mathbf{E}_{T T S}^{r e c}\right)$} \\
\hline Model & Generation & ST & CER (\%) \\
\hline Att MLP-MA & \multirow{2}{*}{ Teacher-forcing } & $\operatorname{argmax}$ & 5.75 \\
\hline Att MLP-MA & & gumbel & 5.7 \\
\hline Att MLP-MA & \multirow{2}{*}{ Greedy } & $\operatorname{argmax}$ & 5.84 \\
\hline Att MLP-MA & & gumbel & 5.88 \\
\hline
\end{tabular}

and the ASR parameters were updated based on the gradient from the sum of the two losses. We have four different scenarios, most of which provide significant improvement compared to the baseline model that is only trained on $\mathrm{Ł}_{A S R}$ loss. With teacherforcing and sampling from Gumbel-softmax, we obtained $11 \%$ relative improvement compared to our best baseline Att MLP-MA.

\section{Related Works}

Approaches that utilize end-to-end feedback learning from source-to-target and vice-versa remain scant. Senrich et al. 22 improved the NMT performance by back-translation on a monolingual dataset. Semisupervised learning for NMT called dual learning [23] was also proposed by combining reconstruction loss and language model reward. However, the feedback gradient provided by the reconstruction loss only limited the closest module to the loss. One primary reason is that the nature of text modalities is represented by discrete variables. Our previous speech chain paper 2, 3 focused on utilizing the closed-loop between ASR and TTS as a semi-supervised learning method. If one of the modalities of data is missing, we can generate a pseudo-pair and train one of the models by reconstruction loss. But, as we described earlier, the study also limit the back-propagation to the closest module due to similar reason that the output of the ASR is discrete tokens. In contrast, in this paper, we successfully address the problem using a straight-through estimator to predict the gradient through discrete variables.

\section{Conclusions}

We introduced a different perspective from a speech chain mechanism. We trained our ASR module by adding feedback from the TTS reconstruction loss. However, the ASR output is not differentiable because of the transcription generated by the discretization process. To address this problem, we used a straight-through estimator to enable the gradient from the TTS module to flow through discrete variables. We tried various scenarios with different decoding and discretization processes. From our experimental results, with teacher-forcing and sampling from Gumbel-Softmax, we improved the ASR performances by $11 \%$ relative CER reduction compared to our baseline.

\section{Acknowledgment}

Part of this work was supported by JSPS KAKENHI Grant Numbers JP17H06101 and JP17K00237.

\section{References}

[1] P. Denes and E. Pinson, The Speech Chain. Anchor books, Worth Publishers, 1993.

[2] A. Tjandra, S. Sakti, and S. Nakamura, "Listening while speaking: Speech chain by deep learning," in 2017 IEEE Automatic Speech Recognition and Understanding Workshop (ASRU), pp. 301-308, Dec 2017.

[3] A. Tjandra, S. Sakti, and S. Nakamura, "Machine speech chain with one-shot speaker adaptation," in Interspeech 2018, 19th Annual Conference of the International Speech Communication Association, Hyderabad, India, 2-6 September 2018., pp. 887-891, 2018.

[4] Y. Bengio, N. Léonard, and A. Courville, "Estimating or propagating gradients through 
stochastic neurons for conditional computation," arXiv preprint arXiv:1308.3432, 2013.

[5] G. Hinton, "Neural networks for machine learning, Coursera video lectures," 2012.

[6] G. Hinton, O. Vinyals, and J. Dean, "Distilling the knowledge in a neural network," arXiv preprint arXiv:1503.02531, 2015.

[7] E. Jang, S. Gu, and B. Poole, "Categorical reparameterization with gumbel-softmax," 2017.

[8] C. J. Maddison, A. Mnih, and Y. W. Teh, "The concrete distribution: A continuous relaxation of discrete random variables," arXiv preprint arXiv:1611.00712, 2016.

[9] D. Bahdanau, K. Cho, and Y. Bengio, "Neural machine translation by jointly learning to align and translate," arXiv preprint arXiv:1409.0473, 2014.

[10] W. Chan, N. Jaitly, Q. Le, and O. Vinyals, "Listen, attend and spell: A neural network for large vocabulary conversational speech recognition," in Acoustics, Speech and Signal Processing (ICASSP), 2016 IEEE International Conference on, pp. 4960-4964, IEEE, 2016.

[11] M.-T. Luong, H. Pham, and C. D. Manning, "Effective approaches to attention-based neural machine translation," arXiv preprint arXiv:1508.04025, 2015.

[12] Y. Wang, R. Skerry-Ryan, D. Stanton, Y. Wu, R. J. Weiss, N. Jaitly, Z. Yang, Y. Xiao, Z. Chen, S. Bengio, et al., "Tacotron: A fully end-to-end text-to-speech synthesis model," arXiv preprint arXiv:1703.10135, 2017.

[13] D. Griffin and J. Lim, "Signal estimation from modified short-time fourier transform," IEEE Transactions on Acoustics, Speech, and Signal Processing, vol. 32, no. 2, pp. 236-243, 1984.

[14] D. B. Paul and J. M. Baker, "The design for the wall street journal-based csr corpus," in Proceedings of the workshop on Speech and Natural
Language, pp. 357-362, Association for Computational Linguistics, 1992.

[15] D. Povey, A. Ghoshal, G. Boulianne, L. Burget, O. Glembek, N. Goel, M. Hannemann, P. Motlicek, Y. Qian, P. Schwarz, J. Silovsky, G. Stemmer, and K. Vesely, "The Kaldi speech recognition toolkit," in IEEE 2011 Workshop on Automatic Speech Recognition and Understanding, IEEE Signal Processing Society, Dec. 2011. IEEE Catalog No.: CFP11SRW-USB.

[16] A. Y. Hannun, A. L. Maas, D. Jurafsky, and A. Y. Ng, "First-pass large vocabulary continuous speech recognition using bi-directional recurrent DNNs," arXiv preprint arXiv:1408.2873, 2014 .

[17] A. Graves, "Supervised sequence labelling," in Supervised Sequence Labelling with Recurrent Neural Networks, pp. 5-13, Springer, 2012.

[18] D. Bahdanau, J. Chorowski, D. Serdyuk, P. Brakel, and Y. Bengio, "End-to-end attention-based large vocabulary speech recognition," in Acoustics, Speech and Signal Processing (ICASSP), 2016 IEEE International Conference on, pp. 4945-4949, IEEE, 2016.

[19] A. Tjandra, S. Sakti, and S. Nakamura, "Multiscale alignment and contextual history for attention mechanism in sequence-to-sequence model," To appear in IEEE SLT 2018, 2018.

[20] S. Kim, T. Hori, and S. Watanabe, "Joint ctc-attention based end-to-end speech recognition using multi-task learning," in Acoustics, Speech and Signal Processing (ICASSP), 2017 IEEE International Conference on, pp. 48354839, IEEE, 2017.

[21] A. Tjandra, S. Sakti, and S. Nakamura, "Attention-based wav2text with feature transfer learning," in 2017 IEEE Automatic Speech Recognition and Understanding Workshop, ASRU 2017, Okinawa, Japan, December 16-20, 2017, pp. 309-315, 2017. 
[22] R. Sennrich, B. Haddow, and A. Birch, "Improving neural machine translation models with monolingual data," in Proceedings of the 54th Annual Meeting of the Association for Computational Linguistics (Volume 1: Long Papers), vol. 1, pp. 86-96, 2016.

[23] D. He, Y. Xia, T. Qin, L. Wang, N. Yu, T. Liu, and W.-Y. Ma, "Dual learning for machine translation," in Advances in Neural Information Processing Systems, pp. 820-828, 2016. 